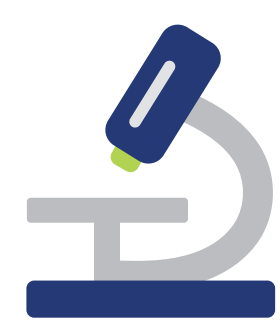

\title{
TRATAMENTO DE SUPERFÍCIES DE TITÂNIO POR OXIDAÇÃO POR PLASMA ELETROLÍTICO PARA USO BIOMÉDICO
}

TREATMENT OF TITANIUM SURFACES BY OXIDATION TO THE ELECTROLYTIC

\author{
PLASMA FOR BIOMEDICAL USE
}

\begin{abstract}
Ana Karenina de Oliveira Paiva
Bacharelado em Ciências e Tecnologia com ênfase em Tecnologia dos Materiais (2014) pela Universidade Federal do Rio Grande do Norte (UFRN), graduação em Engenharia de Materiais (2016) e mestrado em Mestrado Profissional em Ciências, Tecnologia e Inovação (2018) pela UFRN. Contato: kareninapaiva@ outlook.com
\end{abstract}

Arlindo Balbino Nascimento Neto

Graduação em Engenharia Mecânica (2011) pela Universidade Federal do Rio Grande do Norte (UFRN), mestrado em Engenharia Mecânica (2014) e doutorado em Engenharia Mecânica (2018) pela UFRN.

Contato: abnetoengmec@gmail.com

João Marcos Teixeira Lacerda

Graduação em Tecnologia em Desenvolvimento de Software (2008) pelo Instituto Federal de Educação, Ciência e Tecnologia do Rio Grande do Norte, mestrado em Engenharia Elétrica e de Computação (2011) e doutorado em Engenharia Elétrica (2017) pela Universidade Federal do Rio Grande do Norte (UFRN).

Professor de Ensino Básico, Técnico e Tecnológico do IFRN. Contato: joao.lacerda@lais.uol.ufrn.br

Hélio Roberto Hékis

Graduação em Ciências Contábeis (1981) pela Universidade Federal de Santa Catarina (UFSC), mestrado em Administração (1999) pela Universidade do Estado de Santa Catarina (UDESC) e doutorado em

Engenharia de Produção pela UFSC. Professor Associado do Departamento de Engenharia Biomédica da Universidade Federal do Rio Grande do Norte. Contato: hekis1963@gmail.com

João Paulo Queiroz dos Santos

Graduação em Bacharelado em Sistema de Informação (2004) pela Faculdade Natalense para o

Desenvolvimento do RN (FARN), mestrado (2009) e doutorado (2014) em Engenharia Elétrica pela Universidade Federal do Rio Grande do Norte (UFRN). Professor de Ensino Básico, Técnico e Tecnológico do IFRN. Contato: joao.queiroz@lais.ufrn.br

Custódio Leolpodino de Brito Guerra Neto

Graduação em Odontologia (1987) pela Universidade Federal do Rio Grande do Norte (UFRN), mestrado em Engenharia Mecânica (2001) e doutorado em Ciência e Engenharia de Materiais (2005) pela UFRN. Professor Associado da UFRN no Departamento de Engenharia Biomédica. Contato: custodioimplante@gmail.com

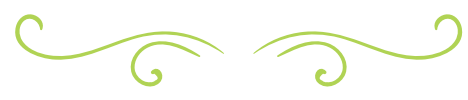




\section{RESUMO}

Os implantes biomédicos surgiram para solucionar diversos problemas na área da saúde, principalmente na reabilitação de pacientes, por meio de de Implantes Odontomédicos, melhorando a qualidade de vida. Na busca por superfícies de implantes que supram a necessidade de obter uma rápida osseointegração em áreas de baixa densidade óssea, inúmeras pesquisas têm sido desenvolvidas para modificar a morfologia superficial. Há vários processos que envolvem métodos mecânicos, químicos e físicos, objetivando os mais variados graus de texturas. Dentre eles existe a Oxidação por Plasma Eletrolítico (Plasma Eletrolytic Oxidation - PEO). Com o intuito de resolver o problema, foi utilizado o PEO como método de tratamento de superfícies. Foram tratados 18 cilindros de titânio grau II, com $3 \mathrm{~mm}$ de diâmetro e $25 \mathrm{~mm}$ de comprimento. O tempo de tratamento utilizado foi de 1, 8 e 16 minutos. A solução eletrolítica estava na temperatura ambiente e a tensão aplicada foi de 290 V. Após os tratamentos, as amostras foram caracterizadas por Fluorescência de Raios-X (FRX), Microscopia Ótica (MO), Microscopia Eletrônica de Varredura (MEV) e por Espectroscopia de Raios-X e Energia Dispersiva (EDS). Em seguida, foi realizada Microscopia de Força Atômica (AFM) para caracterização da textura superficial. Realizou-se ainda a inspeção visual, assim como foram feitos ensaios de molhabilidade. Identificou-se neste trabalho, através das análises químicas pela Fluorescência de Raios-X, a presença do TiO2 na superfície da amostra. Verificou-se no MEV que os revestimentos exibem uma característica porosa, apresentando uma interface bem aderida e sem presença de espaços vazios. Nas imagens do AFM observou-se que com o aumento do tempo de tratamento houve uma maior rugosidade e um aumento da homogeneidade da distribuição dos cristais cerâmicos na superfície. Os ensaios de molhabilidade apresentaram um ângulo de molhamento menor para as amostras com o tratamento por PEO com tempo de 1 e 8 minutos. Conclui-se que a técnica por Oxidação por Plasma Eletrolítico mostrou-se eficaz na deposição de um revestimento cerâmico na superfície da liga de titânio.

Palavras-chave: Tratamento de superfícies. Implantes biomédicos. Titânio. PEO.

\section{ABSTRACT}

Biomedical implants have emerged to solve various health problems, mainly in patient rehabilitation, through dental implants, improving a quality of life. Researches on implant surfaces that provide a need to obtain a rapid osseointegration in areas of low bone density, numerous researches have been developed to modify the surface morphology. There are several processes involving mechanical, chemical and physical methods, aiming at the most varied degrees of textures. Among them, there is an Electrolytic Plasma Oxidation (PEO). In order to solve the problem, PEO was used as a surface treatment method. They were treated with 18 cylinders of grade II titanium, $3 \mathrm{~mm}$ in diameter and $25 \mathrm{~mm}$ in length. The treatment time was 1, 8 and 16 minutes. An electrolytic solution was at room temperature and applied measurement of $290 \mathrm{~V}$. After the treatments, as samples were characterized by X-Ray Fluorescence (FRX), Optical Microscopy (OM), Scanning Electron Microscopy (SEM) and Lightning Spectroscopy $-X$ and Dispersive Energy (EDS). Empute Atomic Force Microscopy (AFM) for surface texture characterization. Visual inspection and wettability tests were also carried out. The presence of $\mathrm{TiO} 2$ on the surface of the sample is identified in the work by means of chemical analysis by X-ray Fluorescence. It was verified in the MEV that the coatings exhibit a porous characteristic, presenting an interface well adhered and without presence of empty spaces. In the images of the AFM it was observed that with the increase of the treatment time 
with a greater roughness and increase of the homogeneity of the distribution of the ceramic crystals in the surface. In the wettability tests they presented a lower wetting angle for samples with PEO treatment with time of 1 and 8 minutes. It is concluded that the Electrolytic Plasma Oxidation technique proved to be effective in the deposition of a ceramic coating on the surface of the titanium alloy.

Keywords: Surface treatment. Biomedical implants. Titanium. PEO.

\section{INTRODUÇÃO}

A aplicação de revestimentos sobre a superfície de metais, para melhorar propriedades como aparência, resistência ao desgaste e corrosão, aderência, entre outras, é uma solução viável e bastante empregada (RAJ; MUBARAK, 2009). Desde a década de 70, os implantes odontomédicos se tornaram a melhor ferramenta para a reabilitação de pacientes. Na odontologia esses implantes foram produzidos para seguir um rígido protocolo cirúrgico que permite o tratamento dos indivíduos com ausências totais ou parciais dos dentes, e continuam sendo utilizados até os dias de hoje. Embora a taxa de sucesso dos implantes dentais seja alta, ainda há ocorrências de falhas. A maioria destas ocorre após a inserção dos implantes no primeiro ano. Atualmente, tem-se buscado uma melhor e rápida osseointegração, visto que os implantes ficam em contato direto com o meio biológico (WISMEYER; VAN WASS; VERMEEREN, 1995).

Para uma melhor osseointegração, muitas tecnologias têm sido desenvolvidas no sentido de potencializar as propriedades químicas, mecânicas e biológicas dessas superfícies, introduzindo uma reparação óssea rápida, guiada, possibilitando uma função duradoura (PULEO; NANCl, 1999). Dentre os biomateriais utilizados, o titânio comercialmente puro (cpTi) e as ligas de titânio com alumínio e vanádio (Ti-Al6-V4) se tornaram os materiais metálicos mais utilizados para a confecção de implantes dentais e ortopédicos, por inúmeras propriedades que esses metais apresentam. Pode-se citar estabilidade química, biocompatibilidade, boas propriedades biomecânicas e formação de óxido superficial bioinerte como características para a escolha de um biomaterial apropriado (PIRES; BIERHALZ; MORAES, 2015). No entanto, eles não formam uma ligação química extremamente satisfatória com o tecido ósseo. Para solucionar isso, os estudos tentam modificar e ainda melhorar suas propriedades de superfície e aumentar o grau de biocompatibilidade do implante em relação ao tecido ósseo para promover uma melhor osseointegração (BECK; LANGE; NEUMANN, 2007; WISMEYER; VAN WASS; VERMEEREN, 1995).

Os implantes, uma vez em contato com o meio biológico, estão submetidos a várias mudanças dinâmicas no seu biolíquido e em suas propriedades superficiais, desencadeando uma sequência de reações que ocorre entre o meio biológico e o biomaterial. Ocorre nesse momento a formação de um "filme de condicionamento" que modula as respostas celulares do hospedeiro. As primeiras moléculas a chegar à superfície do titânio são as de água. Muitas pesquisas apontam que o processo de osseointegração acontece mais rapidamente com a texturização da superfície do implante, explicada por Kasemo (2002), pelo aumento da molhabilidade da superfície pelo líquido biológico e não somente a rugosidade, como se acreditava anteriormente. A molhabilidade superficial influencia proteínas, moléculas e células que chegam posteriormente à água (KASEMO, 2002, SILVA et al., 2013).

O desenvolvimento da interface osso-implante é complexo e envolve numerosos fatores. Estes podem ser relacionados ao implante, como material utilizado, ou relacionados com o meio biológico. Dentre eles podemos citar a forma, topografia e química de superfície, mas também a carga 
mecânica, técnica cirúrgica, e as variáveis do paciente, como estado do leito receptor, quantidade e qualidade óssea (BECKER et al., 2013; PULEO; NANCl, 1999).

Para ocorrer a formação de tecido ósseo é necessário que haja o recrutamento e a proliferação de células precursoras de osteoblastos, que se diferenciarão em osteoblastos produzindo a matriz extracelular não mineralizada, que será, subsequentemente, calcificada. Estes eventos são bastante influenciados por algumas propriedades da superfície dos implantes de Titânio. Essas propriedades são: a composição química, a energia de superfície e a textura da superfície, uma combinação entre topografia e rugosidade (SCHWARTZ; BOYAN, 1994).

Uma das técnicas utilizadas hoje para acelerar a osseointegração é a tecnologia por plasma (DEHNAVI et al., 2013). Este é descrito como um gás contendo espécies neutras e eletricamente carregadas como elétrons, íons positivos, íons negativos, átomos e moléculas produzidos através da aplicação de uma diferença de potencial entre dois eletrodos (ALVES JR et al., 2005; DZHURINSKIY et al., 2015).

Para a obtenção de revestimentos que acelerem e melhorem a adesão de tecido ósseo ao titânio, a oxidação por plasma eletrolítico (Plasma electrolytic oxidation
- PEO) vem sendo bastante empregada. $O$ processo PEO utiliza um meio líquido (eletrólito) e a composição do revestimento pode ser controlada pelo ajuste da composição do eletrólito (SRINIVASAN; BLAWERT; DIETZEL, 2009). Neste trabalho usou-se uma variação desse plasma, o PEO. Esse processo é baseado na geração de um gás ionizado, através de um processo eletroquímico, para deposição de camada cerâmica em Titânio. Como consequência dessa técnica, ocorre uma melhoria na qualidade química e morfológica da superfície, potencializando a osseointegração.

\section{METODOLOGIA}

Foram utilizados neste trabalho 18 cilindros de $\mathrm{Ti}$ cp grau II, com $3 \mathrm{~mm}$ de diâmetro e $25 \mathrm{~mm}$ de comprimento, adquiridos junto à empresa Singular Implants, em Parnamirim/RN. Até o tratamento superficial por Oxidação à Plasma Eletrolítico (PEO) as amostras foram submetidas a vários processos e caracterizadas posteriormente, como apresentado no Fluxograma a seguir (Figura 1), em 3 etapas: a primeira, a preparação das amostras; a segunda, os tratamentos por PEO; e a terceira, as caracterizações das amostras. 


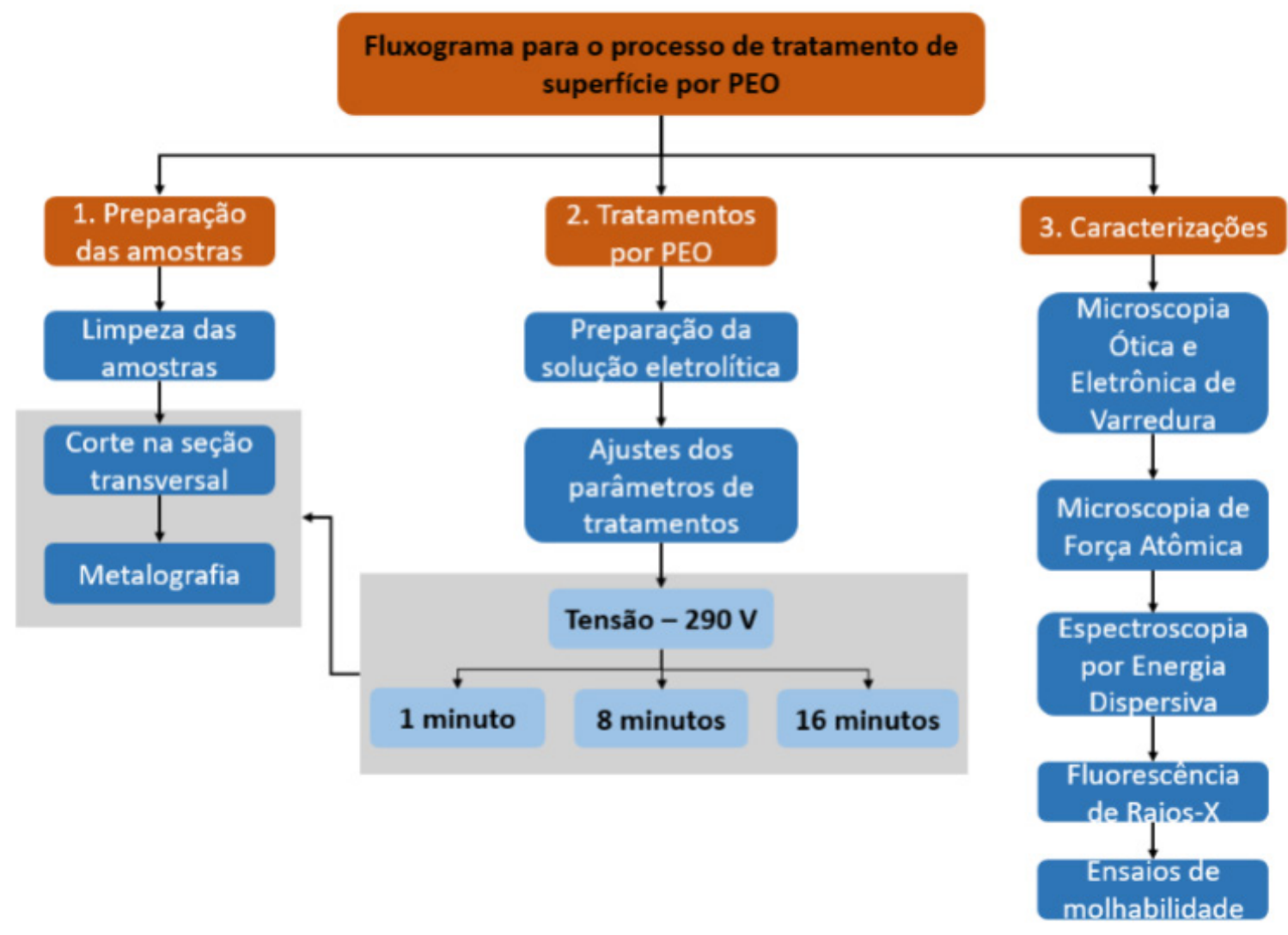

Figura 1 - Fluxograma com o processo para tratamento de superfície por Oxidação por Plasma Eletrolítico. Fonte: autoria própria.

\section{PROCESSO DE LIMPEZA DAS AMOSTRAS}

Os cilindros de Titânio passaram pelo processo rígido de limpeza para eliminar impurezas na superfície que poderiam interferir no processo de oxidação. Através de solução diluída de ácidos fluorídrico (HF) e nítrico (HN), $5 \mathrm{ml}$ de HF em $100 \mathrm{ml}$ de água deionizada e $5 \mathrm{ml}$ de $\mathrm{HN} \mathrm{O}_{3}$ em $100 \mathrm{ml}$ de água destilada, com frações de volume de $10 \%$ e $40 \%$, respectivamente, as amostras ficaram imersas durante 30 segundos para remover a camada de óxido e contaminantes da superfície (WANG et al., 2014). Após essa etapa, as amostras foram limpas em ultrassom (Plana ${ }^{\text {TC }}$ - CBU 100/3L) com acetona e água destilada durante 10 minutos, respectivamente. Em seguida, foi feita a secagem das amostras com secador de ar quente comercial (Taiff Turbo 6000), garantindo a remoção de impurezas que possam contaminar a solução eletrolítica.

\section{PREPARAÇÃO DA SOLUÇÃO ELETROLITITICA}

Com as amostras secas, foram preparados 6 litros de solução eletrolítica nas seguintes proporções dos reagentes: $10 \mathrm{~g} / \mathrm{l}$ de Fosfato de Sódio Tribásico P.A. (TSOP, $\mathrm{Na}_{3} \mathrm{PO} \cdot 12 \mathrm{H} 2 \mathrm{O}$ ) e $2 \mathrm{~g} / \mathrm{l}$ de Hidróxido de potássio $(\mathrm{KOH})$ em 1 litro de água destilada. Foram adicionados $3 \mathrm{~g} / \mathrm{l}$ de Tris Hidroximetil Aminometano $\left(\mathrm{C}_{4} \mathrm{H}_{11} \mathrm{NO}_{3}\right)$ ao eletrólito base como aditivo, para possibilitar um revestimento aderente e favorável à osseointegração (HARIPRASAD et al., 2016). As substâncias citadas foram pesadas em uma balança analítica (Quimis ${ }^{R}$ Q-500L210C) e, posteriormente, foram adicionadas em um béquer de $600 \mathrm{ml}$ e dissolvidas em $400 \mathrm{ml}$ de água destilada. Em seguida, colocou-se a solução em um balão volumétrico de 1 litro, completando-se o volume com água destilada e misturando por 1 minuto. Para cada amostra tratada foram utilizados $600 \mathrm{ml}$ de solução e, para garantir que as 
condições de igualdade de tratamento fossem mantidas, a solução eletrolítica foi trocada a cada experimento.

\section{OXIDAÇÃO POR PLASMA ELETROLÍTICO}

A Figura 2 (a) representa o aparato experimental utilizado para tratar as amostras de Titânio. $O$ equipamento possui três reatores de revestimento PEO, agitador magnético, sistema de recirculação do eletrólito, válvula de controle de vazão e termopar digital Tic 17RGTI (-50 à 105o C), como mostra a Figura 2 (b). As amostras de titânio e o tubo de aço inoxidável foram utilizados como ânodo e cátodo, respectivamente.

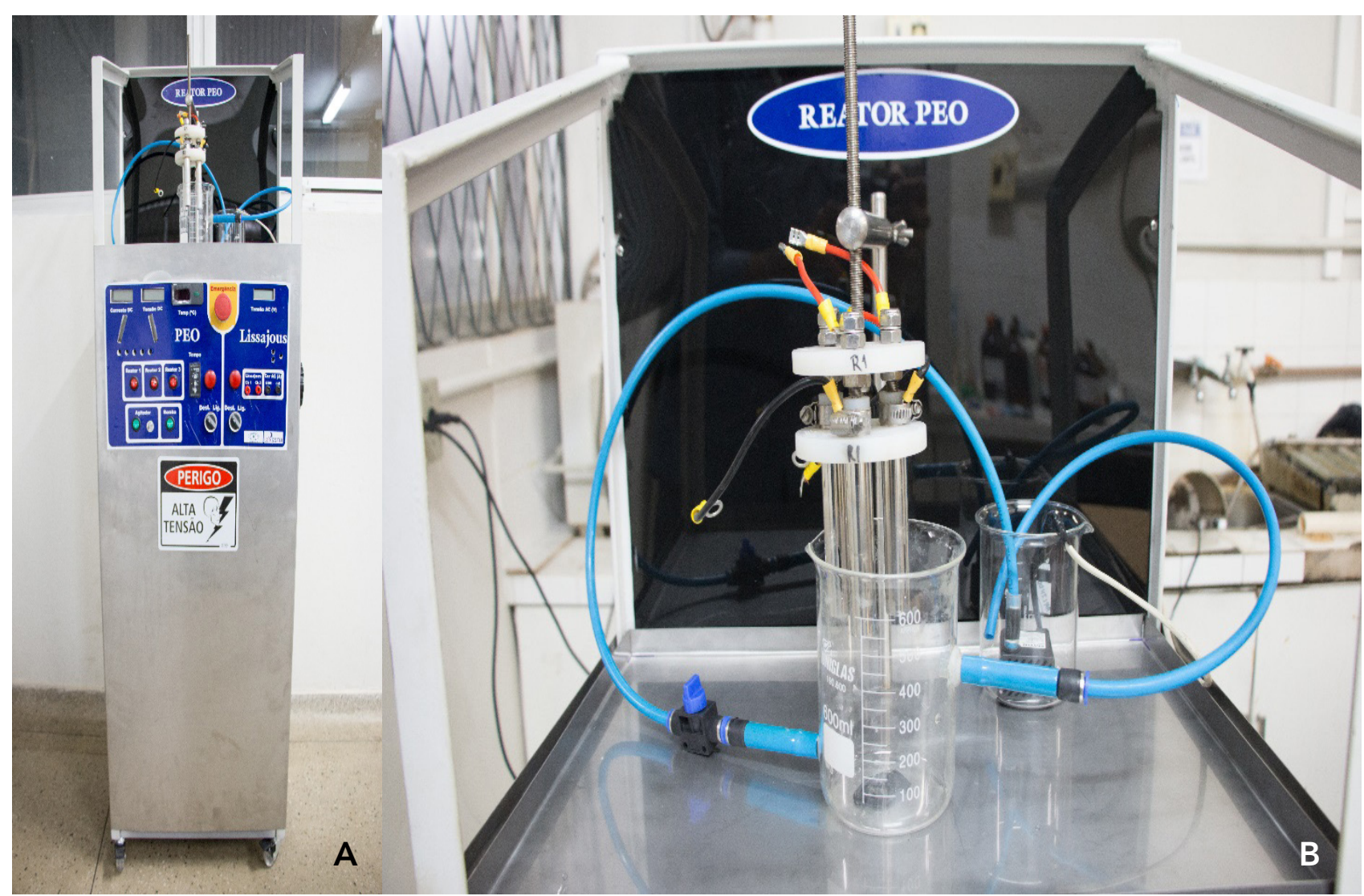

Figura 2 - (a) Equipamento para tratamento por Oxidação por Plasma Eletrolítico (PEO) e (b) Reatores, sistema de controle de vazão e termopar.

Fonte: autoria própria.

As amostras limpas foram colocadas nos reatores do equipamento PEO e imersas em um béquer de $600 \mathrm{ml}$ com $400 \mathrm{ml}$ de solução eletrolítica. Os tratamentos foram realizados nos tempos de $1 \mathrm{~min}, 8 \mathrm{~min}$ e $16 \mathrm{~min}$, submetidos à uma tensão de $290 \mathrm{~V}$ em corrente contínua (CC), escolhida por ser a melhor condição encontrada para este processo. Para cada tempo adotado nesse trabalho, foram realizados três posicionamentos (P1, P2 e P3), dessa forma, utilizando 6 amostras. Portanto, para os três tempos foram utilizadas 18 amostras. A tensão elétrica, a corrente e a temperatura da solução foram monitoradas e registradas a cada minuto. 
PREPARAÇÃO METALOGRÁFICA

Para análise de Microscopia Ótica e Microscopia Eletrônica de Varredura, as amostras após tratamento foram selecionadas para corte em seção transversal, a $4 \mathrm{~mm}$ da extremidade. Após esta etapa, foram embutidas à quente em baquelite, e lixadas com as lixas de carbeto de silício com granulometria 120, 220, 360, 600, 1000 e 1200 mesh, e, por fim, polidas com sílica coloidal composta de $60 \%$ de peróxido de hidrogênio $\left(\mathrm{H}_{2} \mathrm{O}_{2}\right)$ e $40 \%$ de sílica coloidal 0,06 $\mu \mathrm{m}$. Após essa etapa, as superfícies foram limpas com água e acetona e secadas por secador de ar quente comercial.

\section{CARACTERIZAÇÕES}

As amostras foram submetidas às caracterizações de Microscopia Ótica (MO) de luz refletida para análise de espessura dos revestimentos através do software Image Pro Plus. Para análise morfológica da superfície, foram retiradas 5 medidas de espessura de camada para cada amostra (WHEELER et al., 2010). Utilizou-se um microscópio óptico Olympus BX 60M - Japan acoplado a um software Image-Pro Plus versão 4.5.1.22 para o Windows (número serial 41N41000-29998) Copyright 1993- 2002 Media Cybernetics, Inc. A Microscopia Eletrônica de Varredura (MEV), equipamento Shimadzu por elétrons secundários, foi utilizada para análises mais precisas de espessura, com 5 medidas para cada amostra. E, acoplado ao MEV, foi realizada a análise de composição química dos filmes por Espectroscopia de Raios-X e Energia Dispersiva (EDS).

\section{ENSAIOS DE MOLHABILIDADE}

Após o tratamento, as medidas de molhabilidade dos revestimentos foram realizadas por um goniômetro utilizando o software pinacle do Laboratório de Processamento de Materiais por Plasma (LabPlasma) da UFRN. As amostras cilíndricas foram fixadas na horizontal. Foi utilizada uma micropipeta de volume fixo, posicionada perpendicularmente ao plano horizontal das amostras, depositando $5 \mu \mathrm{l}$ de água destilada sobre a superfície em estudo (ALVES et al., 2005). Os valores da molhabilidade correspondem à média aritmética de 3 medidas realizadas após 5 segundos para cada gota depositada na superfície.

\section{RESULTADOS E DISCUSSÕES}

Após os tratamentos por Oxidação por Plasma Eletrolítico, observou-se que o reator realizou revestimento em hastes de titânio com bom desempenho e não apresentou fugas de corrente em lugares indevidos. O equipamento mostrou-se eficaz na deposição de uma camada cerâmica. Foi possível o controle de todos os parâmetros de forma ergonômica, prática, segura, bem como acompanhar as variantes do processo de oxidação. Obteve-se os resultados de tensão, corrente e temperatura da solução eletrolítica perante intervalos de tempo para cada amostra, apresentadas nas Tabelas 1, 2 e 3, para 1, 8 e 16 minutos, respectivamente. Para 1 minuto de tratamento, os intervalos em análise foram a cada 0,5 min; para 8 minutos, foram em um intervalo de 1 min; e para 16 minutos de tratamento, o intervalo de análise foi de $2 \mathrm{~min}$. 
Tabela 1 - Temperatura da solução eletrolítica, tensão e corrente variando a cada 30 segundos para tratamento de 1 minuto.

\begin{tabular}{cccccc}
\hline & Tempo médio $(\mathrm{min})$ & Temperatura $\left({ }^{\circ} \mathrm{C}\right)$ & Tensão $(\mathrm{V})$ & Corrente $(\mathrm{A})$ \\
\cline { 2 - 6 } Amostra com 1 minuto & 0 & 19 & 240 & 0,60 \\
\hline \begin{tabular}{c} 
de tratamento \\
\cline { 2 - 6 }
\end{tabular} & 0,5 & 25 & 240 & 0,50 \\
\hline
\end{tabular}

Tabela 2 - Temperatura da solução eletrolítica, tensão e corrente variando a cada 1 minuto para tratamento de 8 minutos.

\begin{tabular}{ccccc}
\hline & Tempo médio $(\mathrm{min})$ & Temperatura $\left({ }^{\circ} \mathrm{C}\right)$ & Tensão $(\mathrm{V})$ & Corrente $(\mathrm{A})$ \\
\cline { 2 - 5 } & 1 & 31 & 282 & 0,40 \\
\cline { 2 - 5 } & 2 & 34 & 285 & 0,27 \\
\hline \multirow{3}{*}{$\begin{array}{c}\text { Amostra com 8 minutos } \\
\text { de tratamento }\end{array}$} & 3 & 35 & 287 & 0,25 \\
\cline { 2 - 5 } & 4 & 36 & 287 & 0,20 \\
\hline & 5 & 37 & 288 & 0,19 \\
\hline & 6 & 37 & 288 & 0,15 \\
\hline
\end{tabular}

Tabela 3 - Temperatura da solução eletrolítica, tensão e corrente variando a cada 2 minutos para tratamento de 16 minutos.

\begin{tabular}{|c|c|c|cc|}
\hline & Tempo médio $(\mathrm{min})$ & Temperatura $\left(^{\circ} \mathrm{C}\right)$ & Tensão $(\mathrm{V})$ & Corrente $(\mathrm{A})$ \\
\cline { 2 - 5 } & 0 & 25 & 284 & 0,47 \\
\cline { 2 - 5 } & 2 & 30 & 287 & 0,30 \\
\hline $\begin{array}{c}\text { Amostra com 16 minutos } \\
\text { de tratamento }\end{array}$ & 4 & 31 & 286 & 0,16 \\
\cline { 2 - 5 } & 6 & 32 & 286 & 0,14 \\
\cline { 2 - 5 } & 8 & 32 & 287 & 0,11 \\
\hline & 10 & 32 & 287 & 0,09 \\
\hline & 12 & 32 & 288 & 0,07 \\
\hline
\end{tabular}

Observa-se que os resultados estão de acordo com a revisão teórica, ocorrendo a diminuição da corrente com o aumento do tempo de deposição, já que a camada cerâmica formada aumenta a resistência dielétrica, diminuindo a condução e promovendo descarga luminescente e formação do plasma, assim como mostra Parfenov et al. (2015).

Em todos os tempos de tratamento ocorreu o revestimento cerâmico sobre a superfície do titânio. Durante o estágio de anodização geral, forma-se uma película de óxido porosa na superfície da liga de titânio, assim como descrito por Gowthan, Runnellaiappan e Rameshbabu (2016). As amostras apresentaram revestimentos homogêneos e semelhantes, como visto no exemplo de uma amostra com tratamento na Figura 3 (a), em que há uma coloração branca e aparência fosca devido à deposição do óxido. Já a amostra sem tratamento, Figura 3 (b), apresenta-se lisa, aspecto visual normal do titânio. 


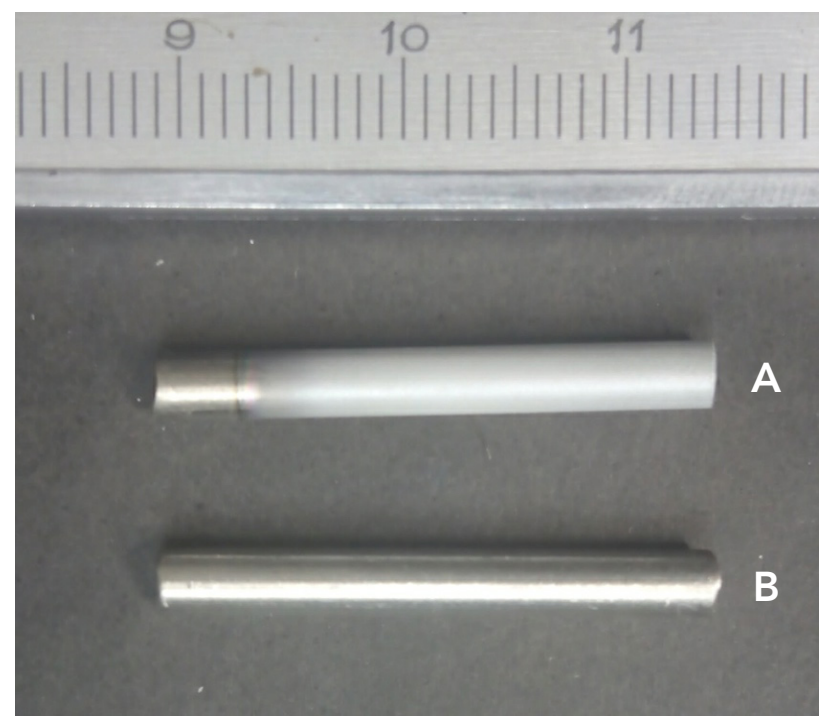

Figura 3 - Hastes de Titânio com tratamento superficial por PEO (a) e sem tratamento (b).

Fonte: autoria própria.

processo por PEO provoca picos de temperatura que fundem os materiais presentes no meio e que, quando são arrefecidos de forma rápida pelo eletrólito, fazem com que o óxido derretido se solidifique na superfície do substrato. Devido ao processo de fusão e solidificação repetidos, induzido pelas descargas, a temperatura permitiu cristalização e transformações da fase do óxido de titânio $\left(\mathrm{TiO}_{2}\right)$ de anatase para rutilo, descrito por Yeung et al. (2013). Também foi identificado neste trabalho, através das análises químicas pela Fluorescência de Raios-X, o $\mathrm{TiO}_{2}$, além dos elementos dos compostos que constituem a solução eletrolítica (TSOP, $\mathrm{Na}_{3} \mathrm{PO}_{4} \cdot 12 \mathrm{H}_{2} \mathrm{O}$ ), $(\mathrm{KOH}), \quad\left(\left(\mathrm{HOCH}_{2}\right) \quad 3 \mathrm{CNH}_{2}\right)$. Como análise complementar, a Espectroscopia de Energia Dispersiva apresentou os elementos presentes na solução e na camada depositada.

Através das análises de Micrografias por Microscópio Ótico e por Microscópio Eletrônico de Varredura (Figuras 4 e 5) pode-se observar a formação da camada cerâmica depositada com eletrólitos da solução eletrolítica. A deposição se deu para todas as amostras entre 1 e 8 minutos, com espessura aproximada de $11 \mu \mathrm{m}$, sendo que com tempo as amostras acima de 8 minutos resultaram na espessura aproximada de $21 \mu \mathrm{m}$. Não houve aumento significativo da espessura de camada quando o tempo ficou acima de 8 minutos de tratamento, devido à alta resistência elétrica.
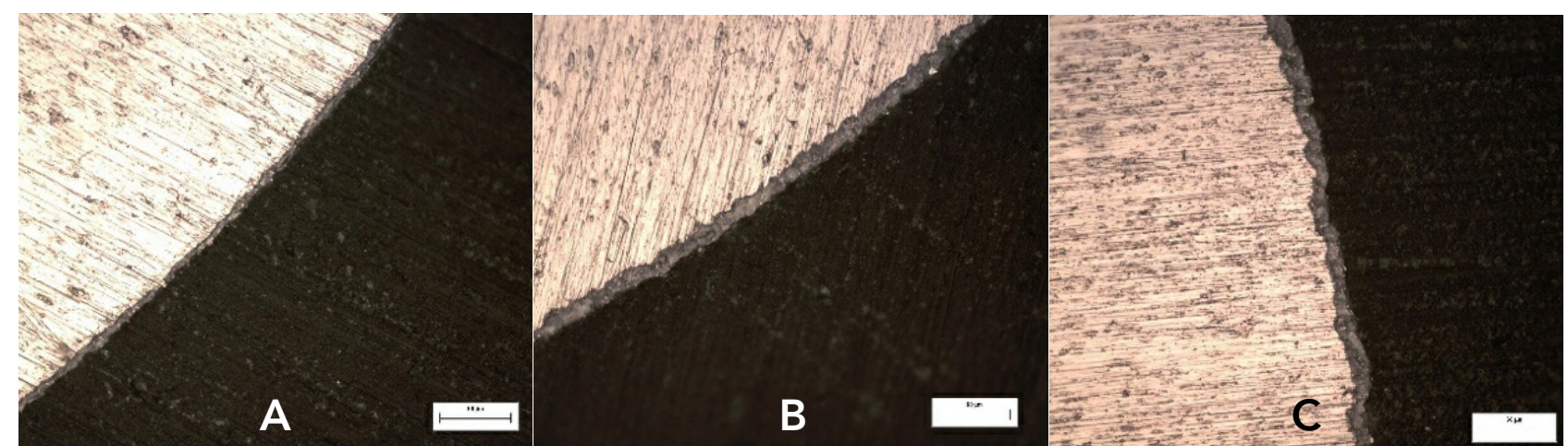

Figura 4 - Micrografias por Microscópio Ótico com aumento de 500 x para: (a) - 1 minuto de tratamento; (b) - 8 minutos de tratamento; (c) - 16 minutos de tratamento.

Fonte: autoria própria. 


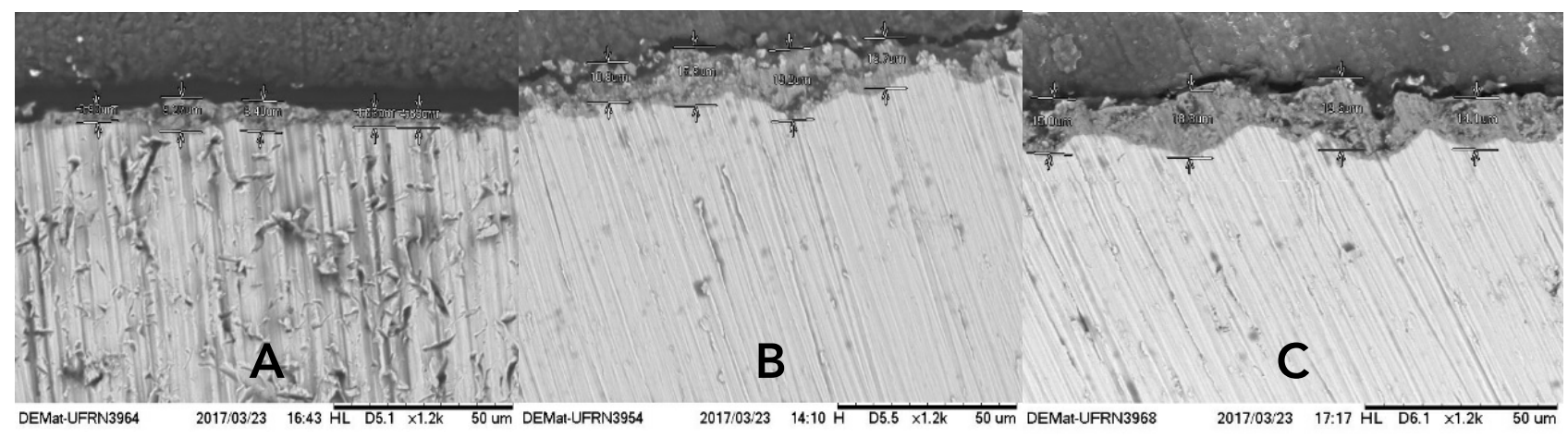

Figura 5 - Micrografias por Microscópio Eletrônico de Varredura com aumento de 1200 x para: (a) - 1 minuto de tratamento; (b) -8 minutos de tratamento; (c) -16 minutos de tratamento.

Fonte: autoria própria.

Todos os revestimentos exibem uma característica comum de um processo PEO, apresentando uma estrutura porosa na camada externa, bem como uma interface Titânio e camada de revestimento, bem aderida e sem presença de espaços vazios, o que propicia uma maior resistência ao desgaste da camada, assim como no estudo realizado por Hariprasad et al. (2016). Com a adição do Tris Hidroximetil Aminometano (C4H11NO3) foi possível promover o aumento da condutividade do eletrólito de forma que diminuiu a resistência dielétrica entre os polos e, consequentemente, aumentou-se a densidade das descargas para o mesmo valor de tensão fornecido que, por sua vez, favoreceu as descargas, resultando numa maior porosidade (BAYATI; MOSHFEGH; GOLESTANI-FARD, 2010).

As imagens de Microscopia de Força Atômica (Figura 6) apresentaram uma variação de rugosidade e textura pela deposição cerâmica, que viabiliza a propriedade de molhabilidade na superfície. Observa-se que com o aumento do tempo de tratamento houve uma maior rugosidade e aumento da homogeneidade da distribuição dos cristais cerâmicos na superfície.

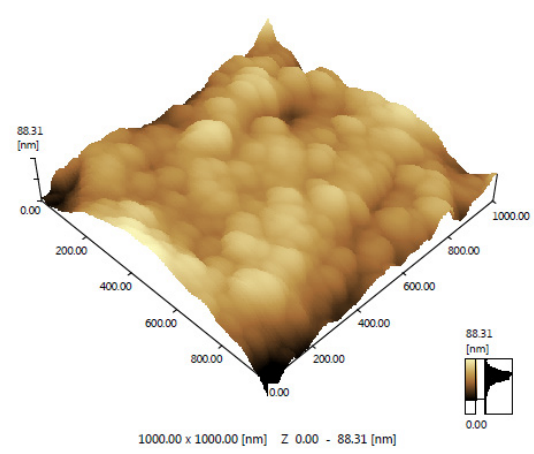

A

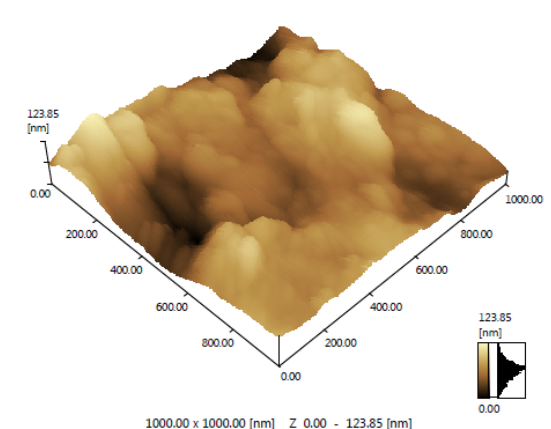

B

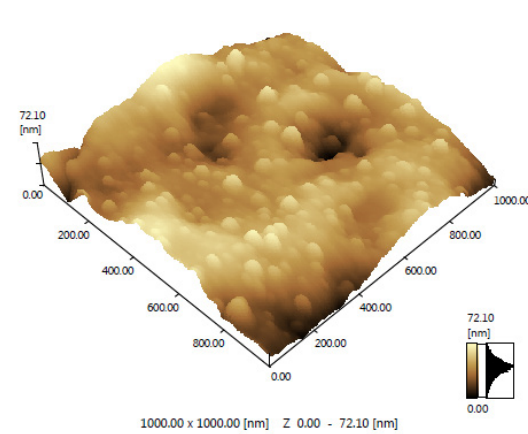

C

Figura 6 - Micrografias por Microscópio de Força Atômica para: (a) - 1 minuto de tratamento; (b) - 8 minutos de tratamento; (c) - 16 minutos de tratamento.

Fonte: autoria própria. 
Bayati, Moshfegh e Golestani-Fard (2010) descrevem que, com o aumento da tensão elétrica no tratamento, um filme é formado em toda a superfície e simultaneamente novas camadas se desenvolvem de forma paralela, ocupando mais da superfície à medida que o tempo de tratamento aumenta. A deposição cerâmica (formação da camada de TiO2) e compostos orgânicos ocorreram de forma gradativa com o aumento do tempo, porém as descargas foram diminuindo acima de 8 minutos, pelo aumento da camada cerâmica, que por sua vez é mais isolante do que a haste de Titânio. Observam-se nas imagens de AFM estas superfícies com grãos arredondados para este tipo de deposição, com uma morfologia propícia à formação de poros.

Os testes de molhabilidade apresentaram uma diminuição significativa do ângulo de molhamento para as amostras com o tratamento por PEO, mudança mais evidente nos tempos de 8 e 16 minutos de tratamento (Tabela 4 e Figura 7).

Tabela 4 - Teste de molhabilidade para amostra sem tratamento e para 1, 8 e 16 minutos de tratamento por PEO.

\begin{tabular}{|c|l|c|}
\hline $\mathbf{N}^{\circ}$ & \multicolumn{1}{|c|}{ Amostra } & $\begin{array}{c}\text { Ângulo de } \\
\text { molhamento }\left(^{\circ}\right)\end{array}$ \\
\hline 1 & Sem tratamento & 42 \\
\hline 2 & Com 1 min de tratamento & 30 \\
\hline 3 & Com 8 min de tratamento & 12 \\
\hline 4 & $\begin{array}{l}\text { Com 16 min de } \\
\text { tratamento }\end{array}$ & 9 \\
\hline
\end{tabular}

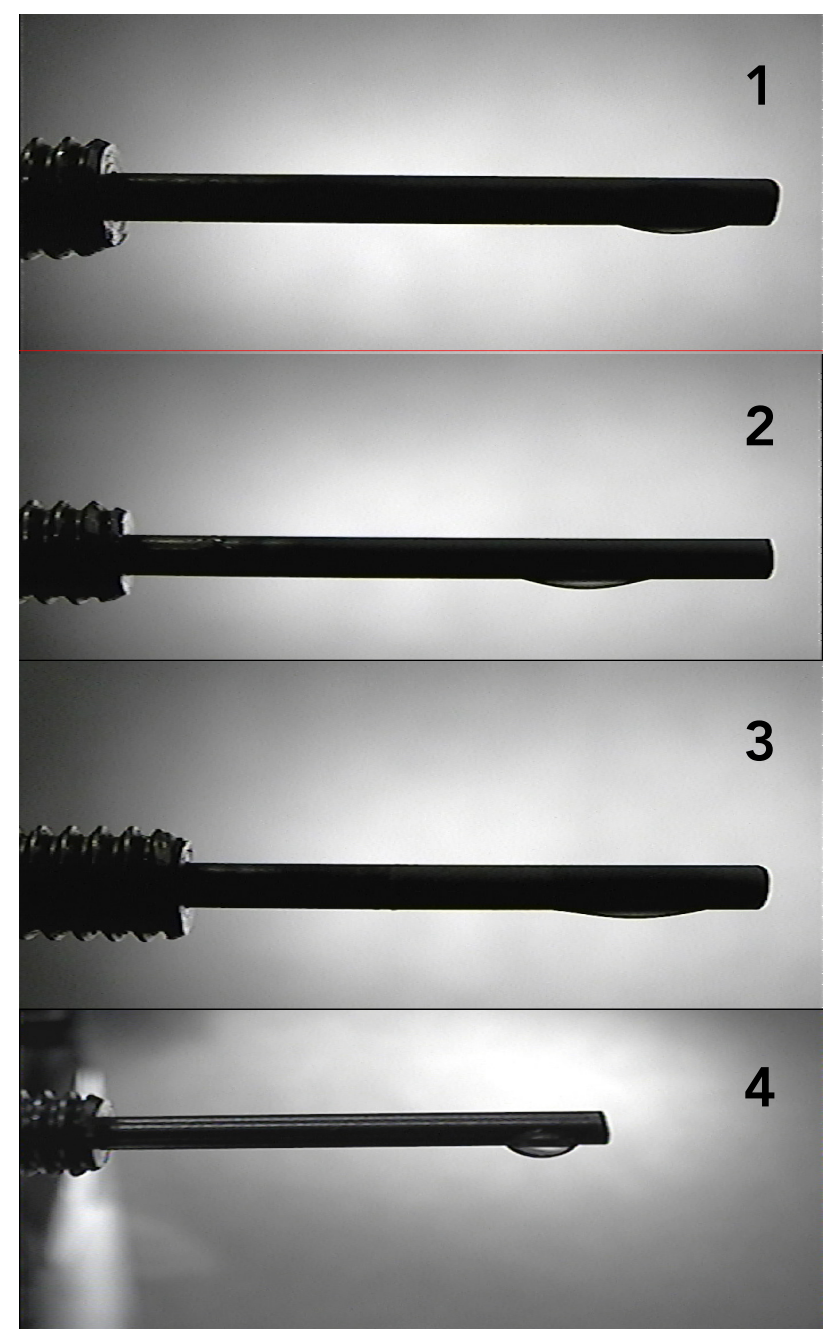

Figura 7 - Análise de molhabilidade de gota pendente: (1) - Sem tratamento; (2) - Tratamento por 1 min; (3) Tratamento por $8 \mathrm{~min}$; (4) - Tratamento por $16 \mathrm{~min}$.

Fonte: autoria própria.

Segundo os pesquisadores Gowtham, Arunnellaiappan e Rameshbabu (2016), uma superfície hidrofílica apresenta-se como um fator necessário para mostrar bioatividade favorável. Os ensaios de molhabilidade apresentaram resultados muito promissores em questão de boa molhabilidade para a superfície cerâmica depositada. Comparando com a literatura, quanto mais o ângulo de molhamento for próximo aos 180 graus, mais molhável é a superfície, fator necessário para mostrar bioatividade favorável, favorecendo a superfície para osseointegração. Observou-se que, com o aumento do tempo de tratamento acima de 8, maior foi o ângulo de molhamento, podendo ser explicado pala grande presença de porosidade 
e rugosidade na superfície consequente do revestimento por $\mathrm{PEO}$.

Wheeler et al (2010) revelaram que revestimento de eletrólito contendo fosfato apresenta um grau de porosidade em maior escala na sua superfície. Dessa forma, todas as condições realizadas obtiveram resultados de ângulos de contato maiores do que a amostra de referência (sem tratamento). Esses resultados indicam que os revestimentos $\mathrm{PEO}$ produzem superfícies hidrofílicas. Isso pode ser explicado pelo aumento da porosidade com o aumento do tempo de tratamento.

\section{CONSIDERAÇÕES FINAIS}

Baseado no que foi exposto, é pertinente afirmar que a técnica por Oxidação por Plasma Eletrolítico mostrou-se eficaz na deposição de uma camada cerâmica na superfície da liga de titânio. Foi possível o controle de todos os parâmetros de forma ergonômica, prática e segura, bem como o acompanhamento das variantes do processo de oxidação. Identificou-se através das análises químicas pela Fluorescência de Raios-X a presença do TiO2 na superfície da amostra. Em análise complementar, a Espectroscopia de Raios-X por Energia Dispersiva apresentou os elementos presentes na solução e na camada depositada.

Através das análises por Microscópio Ótico e por Microscópio Eletrônico de Varredura pode-se observar uma deposição para todas as amostras para 1 minuto com espessura aproximada de $11 \mu \mathrm{m}$. E para os tempos de 8 e 16 minutos, observou-se uma espessura aproximada de $21 \mu \mathrm{m}$. Nas imagens de $M E V$, os revestimentos exibem uma interface sugestiva de boa aderência sem presença de espaços vazios. As imagens de Microscopia de Força Atômica mostraram que com o aumento do tempo de tratamento de 1 para 8 minutos houve uma maior rugosidade e aumento da homogeneidade da distribuição dos cristais cerâmicos na superfície. Os ensaios de molhabilidade apresentaram um ângulo de molhamento menor para as amostras com o tratamento por PEO para os tempos de 8 e 16 minutos.

Podemos concluir que a técnica de tratamento superficial utilizada se apresentou como viável para deposição em superfícies cilíndricas de Titânio, com boas propriedades para possível aplicação em implantes biomédicos, e com bons indícios para os aspectos biomecânicos e biológicos. 


\section{REFERÊNCIAS}

ALVES JR, C. et al. Nitriding of titanium disks and industrial dental implants using hollow cathode discharge. Surface and Coatings Technology, v. 194, p. 196-202, 2005.

BAYATI, M. R.; MOSHFEGH, A. Z.; GOLESTANI-FARD, F. Effect of electrical parameters on morphology, chemical composition, and photoactivity of the nano-porous titania layers synthesized by pulsemicroarc oxidation. Electrochimica Acta, v. 55, n. 8, p. 2760-2766, 2010. ISSN 0013-4686.

BECK, U.; LANGE, R.; NEUMANN, H. G. Micro-plasma textured Ti implant surfaces. Biomolecular Engineering, v. 24, n. 1, p. 47-51, 2007. ISSN 1389-0344.

BECKER, W. et al. Survival rates and bone level changes around porous oxide coated implants (tiunite). Clinical implant dentistry and related research, [s.I.]: Wiley Online Library, v. 15, n. 5, p. 654660, 2013.

DEHNAVI, V. et al. Effect of duty cycle and applied current frequency on plasma electrolytic oxidation (PEO) coating growth behavior. Surface and Coatings Technology, v. 226, p. 100-107, 2013. ISSN 0257-8972.

DZHURINSKIY, D. et al. Characterization and corrosion evaluation of TiO2:n-HA coatings on titanium alloy formed by plasma electrolytic oxidation. Surface and Coatings Technology, v. 269, p. 258-265, 2015. Supplement C.

GOWTHAM, S.; ARUNNELLAIAPPAN, T.; RAMESHBABU, N. An Investigation on Pulsed DC Plasma Electrolytic Oxidation of cp-Ti and its Corrosion Behaviour in Simulated Body Fluid. Surf. Coat. Technol., v. 301, p. 63-73, 2016.

HARIPRASAD, S. et al. Role of electrolyte additives on in-vitro corrosion behavior of DC plasma electrolytic oxidization coatings formed on Cp-Ti. Surface and Coatings Technology, v. 292, p. 20-29, 2016. ISSN 0257-8972.

HARIPRASAD, S. et al. Role of electrolyte additives on in-vitro corrosion behavior of DC plasma electrolytic oxidization coatings formed on Cp-Ti. Surface and Coatings Technology, v. 292, p. 20-29, 2016. ISSN 0257-8972.

KASEMO, B. Biological Surface Science. Surface Science, v. 500, p. 656, 2002. 
PARFENOV, E. V. et al. Towards smart electrolytic plasma technologies: An overview of methodological approaches to process modelling. Surface and Coatings Technology, v. 269, p. 2-22, 2015. Supplement C.

PIRES, A. L. R.; BIERHALZ, A. C. K.; MORAES, A. M.; Biomateriais: Tipos, aplicações e mercado. Química Nova, v. 38, n. 7, p. 957971, 2015.

PULEO, D. A.; NANCl, A. Understanding and controlling the boneimplant interface. Biomaterials, v.2 0, n. 23-24, p. 2311-2321, 1999.

RAJ, V.; MUBARAK ALI, M. Formation of ceramic alumina nanocomposite coatings on aluminium for enhanced corrosion resistance. Journal of Materials Processing Technology, v. 209, p. 5341-5352, 2009.

SCHWARTZ, Z.; BOYAN, B.D. Underlying mechanisms at the bonebiomaterial interface. J. Cell Biochem., v. 56, n. 3, p. 340-347, 1994.

SILVA, M. A. M. et al. Influencie of topography on plasma treated titanium surface wettability. Surface and Coatings Technology, v. 235, p. 447-453, 2013.

SRINIVASAN, P. B.; BLAWERT, C.; DIETZEL, W. Dry sliding wear behaviour of plasma electrolytic oxidation coated AZ91 cast magnesium alloy. Wear, v. 266, p. 1241-1247, 2009.

WANG, Y. et al. Preparation and properties of plasma electrolytic oxidation coating on sandblasted pure titanium by a combination treatment. Materials Science and Engineering: C, v. 42, p. 657-664, 2014. Supplement C.

WHEELER, J. M. et al. Evaluation of micromechanical behaviour of plasma electrolytic oxidation (PEO) coatings on Ti-6Al-4V. Surface and Coatings Technology, v. 204, n. 21, p. 3399-3409, 2010.

WISMEYER, D.; VAN WASS, M.; VERMEEREN, J. I. Overdentures supported by ITI implants: A 6,5-year evaluation of patient satisfaction and prosthetic aftercare. Int. J. Oral Maxillofac. Impl., v. 10, n.6, p.744-749, 1995.

YEUNG, W. K. et al. In vitro biological response of plasma electrolytically oxidized and plasma-sprayed hydroxyapatite coatings on Ti-6Al-4V alloy. Journal of Biomedical Materials Research Part B: Applied Biomaterials, v. 101B, n. 6, p. 939-949, 2013. ISSN 1552-4981. 